Proceedings of the

Combustion Institute

\title{
Laminar and unstable burning velocities and Markstein lengths of hydrogen-air mixtures at engine-like conditions
}

\author{
S. Verhelst ${ }^{\mathrm{a}, *}$, R. Woolley ${ }^{\mathrm{b}}$, M. Lawes ${ }^{\mathrm{b}}$, R. Sierens ${ }^{\mathrm{a}}$ \\ ${ }^{a}$ Department of Flow, Heat and Combustion Mechanics, Ghent University, Belgium \\ ${ }^{\mathrm{b}}$ School of Mechanical Engineering, University of Leeds, Leeds LS2 9JT, UK
}

\section{Abstract}

Hydrogen offers an attractive alternative to conventional fuels for use in spark ignition engines. It can be burned over a very wide range of equivalence ratios and with considerable exhaust gas recirculation. These help to minimise pumping losses through throttleless operation and oxides of nitrogen $\left(\mathrm{NO}_{x}\right)$ production through reduced temperature. Full understanding of hydrogen-fuelled engine operation requires data on the laminar burning rate of hydrogen-air residuals under a wide range of conditions. However, such data are sparse. The present work addresses this need for experimental data. Spherically expanding $\mathrm{H}_{2}$-air flames were measured at a range of temperatures, pressures, and equivalence ratios and with varying concentrations of residuals of combustion. Unstretched burning velocities, $u_{1}$, and Markstein lengths, $L_{\mathrm{b}}$, were determined from stable flames. At the higher pressures, hydrodynamic and diffusional-thermal instabilities caused the flames to be cellular from inception and prohibited the derivation of values of $u_{1}$ and $L_{\mathrm{b}}$. The effect of pressure on the burning rate was demonstrated to have opposing trends when comparing stoichiometric and lean mixtures. The present measurements were compared with those available in the literature, and discrepancies were attributed to neglect, in some works, the effects of stretch and instabilities. From the present measurements, the effects of pressure, temperature, and residual gas concentration on burning velocity are quantified for use in a first step towards a general correlation. (c) 2004 by the Combustion Institute. Published by Elsevier Inc. All rights reserved.

Keywords: Hydrogen; Laminar burning velocity; Residuals; Engine

\section{Introduction}

Hydrogen is a very attractive alternative to traditional fossil fuels as an energy carrier due to its very clean combustion and the ease of manufacture. Because of its high flame speed, leading to near constant volume combustion, and wide flam-

\footnotetext{
* Corresponding author. Fax: +3292643590.

E-mail address: sebastian.verhelst@ugent.be (S.
}

mability limits, a hydrogen-fuelled engine has the potential for high efficiency. The power output of such engines can be varied by changing the equivalence ratio to use very lean mixtures at low loads. Oxides of nitrogen are minimised, while maintaining adequate power, by varying the amount of EGR during stoichiometric operation [1]. In both cases, the throttle valve is not used, except maybe at idling, and pumping losses are minimised. Thus, hydrogen engines use a large range of equivalence ratios, and EGR concentrations can be very high with stoichiometric operation. 
Two of the authors have a history in experimental research on hydrogen engines (e.g., [2,3]). Although much knowledge has been gathered from engine tests, they have proved expensive both in money and time. Therefore, a mathematical model to simulate the combustion of hydrogen in spark ignition (SI) engines is being developed at Ghent [4]. This models the influence of the turbulence on the initially laminar flame propagation and assumes quasi-one-dimensional combustion. Such models rely on a known laminar burning velocity at the instantaneous cylinder pressure, temperature, and mixture composition, and they calculate the change in flame speed due to turbulence. A convenient way of obtaining the laminar burning velocity is by a laminar burning velocity correlation. However, the few data that exist on hydrogen-air laminar burning velocities at engine-like pressures and temperatures do not take stretch or cellularity effects into account, causing a large spread in the reported burning velocities [5-8]. Some unstretched burning velocities have been measured but were generally performed at atmospheric conditions [9-15]. Therefore, this paper presents and discusses measurements of the laminar burning velocity, and the effects of flame stretch rate on hydrogen-air and hydrogen-air residual mixtures. For conditions at which measurements of the laminar burning velocity are not possible due to fundamental flame instabilities, measurements of unstable burning velocities, obtained under initially quiescent conditions, are presented.

Using these data, the effects of pressure, temperature, and residual gas concentration on burning velocity are quantified for use in a tentative correlation.

\section{Experimental apparatus and technique}

A $380 \mathrm{~mm}$ diameter, spherical, stainless steel vessel (bomb) was employed which is capable of withstanding the temperatures and pressures generated from explosions with initial pressures of up to $1.5 \mathrm{MPa}$ and initial temperatures of up to $600 \mathrm{~K}$ [16]. It has extensive optical access through three pairs of orthogonal windows of $150 \mathrm{~mm}$ diameter and is equipped with four fans driven by electric motors, used here only to ensure the reactants were well mixed. The fans were switched off prior to ignition allowing sufficient time for any mixture motion to decay. Mixtures were prepared in situ using the partial pressure method in which simulated residuals were synthesised from water and nitrogen and were injected into the bomb starting from $5 \%$ of vacuum, water first. The bomb was then filled to the desired pressure with high purity hydrogen and dry air from a cylinder. Following an explosion, the vessel was first flushed twice with dry air to remove any residual products before a subsequent mixture was prepared. All other aspects of the equipment and experimental technique are given in $[16,17]$.

Following central spark ignition, flame images were captured by schlieren cine' photography using a Phantom V4.1 digital camera at various resolutions and frame rates, depending on the burning rate of the mixture. For the slower flames, a frame rate of 1000 frames per second (fps) was used at the maximum available resolution of $512 \times 512$ pixels. Flame speeds, $S_{n}(=\mathrm{d} r / \mathrm{ddt})$, were then calculated from the mean flame radii, $r$, obtained from measurements of flame projected area. Because of the very high flame speeds obtained with hydrogen, $1000 \mathrm{fps}$ proved to be too slow for the faster burning mixtures. Therefore, higher frame rates of up to $14,300 \mathrm{fps}$ were achieved by capturing only a portion of the flame image at the full 512 pixel width but with a reduced vertical field of view of down to 32 pixels, which corresponded to a field of view of about $150 \mathrm{~mm}$ wide by $9.4 \mathrm{~mm}$ high. Because of the high flame speeds, the flames were almost spherical so there was very little loss in accuracy when using the reduced vertical field of view. Moreover, previous studies with other fuels showed essentially identical flame speeds at all radial locations [17].

The unstretched flame speed, $S_{\mathrm{s}}$, unstretched laminar burning velocity, $u_{1}$, onset of instabilities, and the effect of flame stretch rate, $\alpha$, embodied in the Markstein length were derived from flame speed using the methodology described in [1618]. The total stretch rate on a stable, non-cellular, outwardly propagating flame is given by $\alpha=2$. $S_{n}$ I $r$ [18], and a linear relationship between this and $S_{n}$ is quantified by a burned gas Markstein length, $L_{\mathrm{b}}$, such that $S_{\mathrm{s}}-S_{n}=L_{\mathrm{b}} \alpha . S_{\mathrm{s}}$ is obtained as the intercept value of $S_{n}$ at $\alpha=0$ in the plot of $S_{n}$ against $\alpha$. The unstretched laminar burning velocity is then obtained from $u_{1}=S_{\mathrm{s}} /\left(\rho_{\mathrm{u}} / \rho_{\mathrm{b}}\right)$, where $\rho$ is the density, and the subscripts ' $u$ ' and ' $b$ ' refer to the unburned and equilibrated adiabatic products.

A problem arises with the above technique when a flame becomes unstable leading to a cellular flame structure. Under such conditions, the flame accelerates as the stretch rate reduces, and a linear relationship between $S_{n}$ and $\alpha$ no longer applies. The methodology adopted in [17-19] was to extrapolate only the linear portion of the $S_{n}$ against $\alpha$ curve to zero. This approach relies on there being a sufficiently large stable, linear, regime. Further, it has been shown that the onset of flame acceleration due to cellularity occurs at a critical Peclet number, $P e_{\mathrm{cl}}=r_{\mathrm{cl}} / \delta_{1}$ [19], where $r_{\mathrm{cl}}$ is the radius at which cells start to induce flame acceleration, and $\delta_{1}$ is the laminar flame thickness. This increases linearly with Markstein number, $M a_{\mathrm{b}}=L_{\mathrm{b}} / \delta_{1}$. Because the hydrogen flames used in the present work have a very thin reaction zone, the critical Peclet number is attained at small flame radii and, hence, only for mixtures at 
1651 bar were reliable extrapolations to zero stretch

166 rate possible. The higher pressure flames were cel-

167 lular from the first recorded flame image which

168 prohibited the derivation of $u_{1}$ and $L_{\mathrm{b}}$. The range

169 of conditions at which stable hydrogen-fuelled

170 flames existed was much lower than observed with

171 both methane [19] and iso-octane [17]. Therefore, to study the influence of temperature, pressure, and residual gas content, the flame speed at a flame radius of $10 \mathrm{~mm}$ was recorded, $S_{n, 10 \mathrm{~mm}}$, and divided by the density ratio $\rho_{\mathrm{u}} / \rho_{\mathrm{b}}$ to yield a burning velocity, $u_{n, 10 \mathrm{~mm}}$. This burning velocity is not a fundamental parameter, but is indicative of the burning rate at a fixed condition. It represents a compromise that involves a sufficiently large radius to minimise the effects of the spark, while being small enough to limit the acceleration due to the instabilities. An alternative strategy, although not representative of engine conditions, would be to replace the nitrogen in the mixture with helium, resulting in a stable flame from which the unstretched laminar burning velocity could be measured [20,21].

\section{Results and discussion}

\subsection{Flame speed measurements}

Conditions that correspond to those in a typical engine, including stoichiometric mixtures with a wide range of residual concentrations and a wide range of lean mixtures with low concentrations of residuals, were studied. At least two measurements were performed at each condition, and the repeatability was generally excellent. In most cases uncertainties were within $5 \%$.

Shown in Fig. 1 is the variation of flame speed with radius for flames at an initial pressure of 1 bar, $\phi=1.0$ and temperatures of 300,365 , and $430 \mathrm{~K}$. In each case, the flame speed increases with radius, and flame images show that they are smooth throughout most of the filming period. The increasing temperature results in a corresponding increase in the flame speed. However, apart from the overall magnitude of the flame

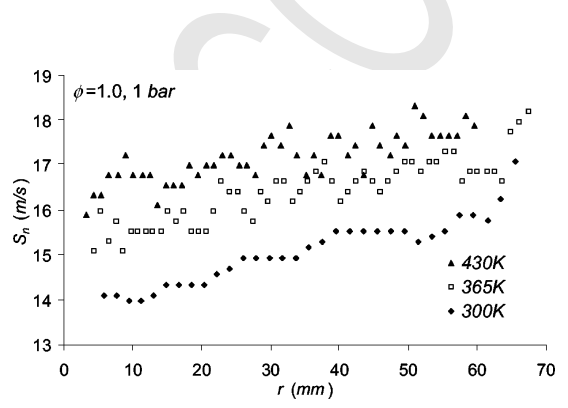

Fig. 1. Flame speed vs. flame radius for a $\phi=1,1$ bar flame at various temperatures.

speed there appears little influence of temperature on flame behaviour. Shown in Fig. 2 is the variation of flame speed with radius for flames at the same conditions as those in Fig. 1, but at $\phi$ $=0.5$. Corresponding flame images for a flame at $\phi=0.5$ and $365 \mathrm{~K}$ are shown in Fig. 3. Here, the flame surface is initially smooth and, in contrast with the stoichiometric flames, the flame speed reduces with radius. However, after about $20 \mathrm{~mm}$ (corresponding to $P e_{\mathrm{cl}}=672$ for the 300 and $430 \mathrm{~K}$ flames and $P e_{\mathrm{cl}}=614$ for the $365 \mathrm{~K}$ flame), the flame surface becomes cellular and there is a corresponding increase in the flame speed. This reduction, followed by acceleration due to cellularity in the flame speed with radius is the same for all three flames in Fig. 2.

The effect of pressure on the flame speed of stoichiometric flames at an initial temperature of $365 \mathrm{~K}$ is shown in Fig. 4. The increase in flame speed with radius is much greater for the flames at 5 and 10 bar than for the one at 1 bar. This corresponds to a difference in flame structure. As discussed above, the flame at 1 bar was smooth throughout the flame growth, whereas those at 5 and 10 bar were cellular throughout, as illustrated by the flame images in Fig. 5 for the flame at 5 bar, and a consequence of the reduced flame thickness at higher pressures. Shown in Fig. 6 is the variation of flame speed with radius and pres-

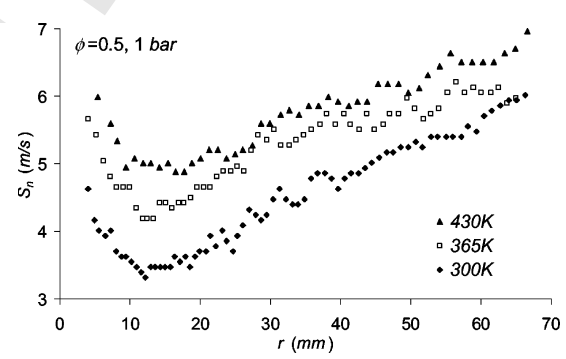

Fig. 2. Flame speed vs. flame radius for a $\phi=0.5,1$ bar flame at various temperatures.

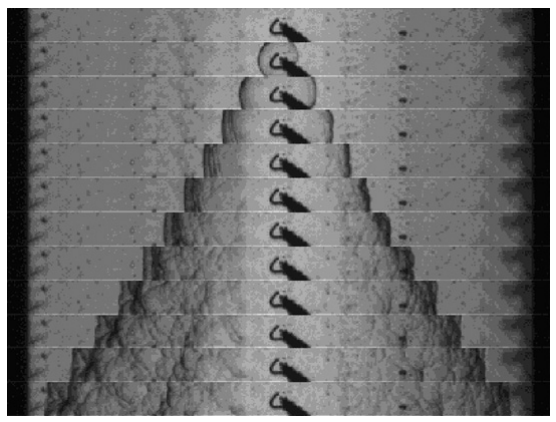

Fig. 3. Development of a $\mathrm{H}_{2}$-air flame at $\phi=0.5$, $365 \mathrm{~K}$, and 1 bar, time between frames $=1.2 \mathrm{~ms}$. 


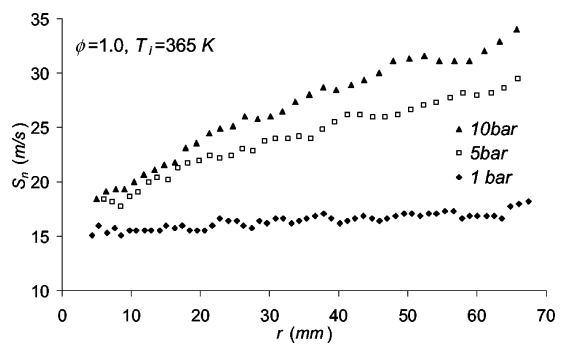

Fig. 4. Flame speed vs. flame radius for a flame at $\phi=1$ and $365 \mathrm{~K}$ at various pressures.

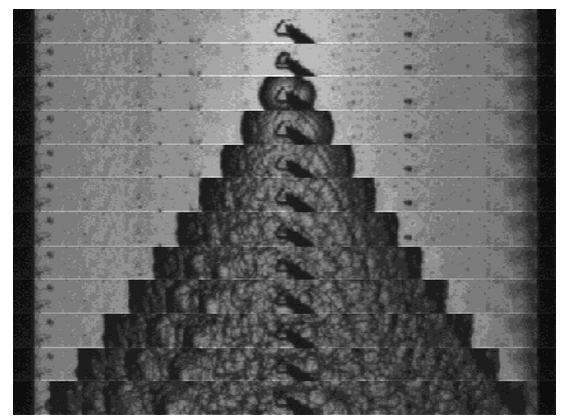

Fig. 5. Development of a flame at $\phi=1,365 \mathrm{~K}$, and 5 bar, time interval: $0.28 \mathrm{~ms}$.

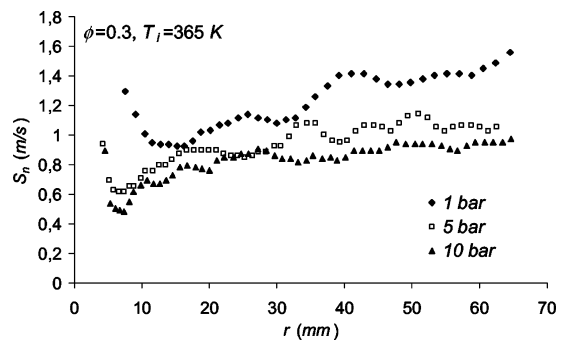

Fig. 6. Flame speed vs. flame radius for a $\phi=0.3,365 \mathrm{~K}$ flame at various pressures.

sure for lean flames at $\phi=0.3$ and $365 \mathrm{~K}$. Each

\section{curve exhibits the same trend as those in Fig. 2} in that there is an initial reduction, followed by an acceleration in the flame speed as cells develop. Similar to stoichiometric flames, the propensity to cellularity is greater as the pressure is increased. This is indicated in Fig. 6 by an earlier onset of acceleration with increasing pressure. However, the effect of pressure on the flame speed is the opposite of that observed for stoichiometric flames.

The effect of residual gas concentration on the flame speed of flames at 1 bar, $365 \mathrm{~K}$, and $\phi=0.8$ is shown in Fig. 7 for residual gas concentrations of $0,10,20$, and $30 \mathrm{vol} \%$. Increasing residual gas concentration causes a reduction in flame speed and an earlier transition to cellularity. Hence,

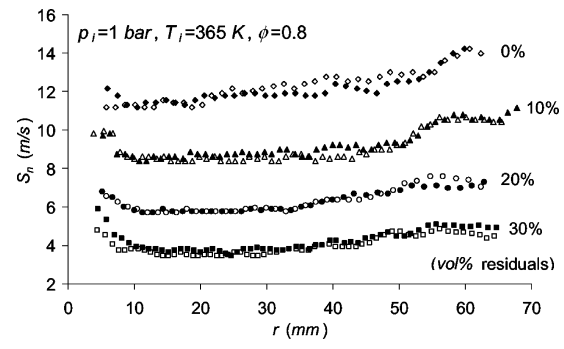

Fig. 7. Flame speed vs. flame radius for flames at $\phi$ $=0.8,365 \mathrm{~K}$, and 1 bar, with varying residual gas concentrations.

diluting with residuals has the same effect on the 253 flame speed and stabilities (cells) as does dilution 254 with air.

\subsection{Burning velocity and Markstein length}

Shown by the symbols in Fig. 8 is the effect of residual gas concentration, $f$, on the variation of flame speed with flame stretch rate for flames at 1 bar, $365 \mathrm{~K}$, and $\phi=0.8$. It was obtained from the data in Fig. 7. Extrapolation of these data to zero stretch rate, as shown by the solid lines, yields the unstretched laminar burning velocity and Markstein length as shown in Figs. 9 and 10 for a range of equivalence ratios. At all values of $f$, the Markstein length increases with $\phi$. However, all values of $L_{\mathrm{b}}$ remain negative except those for the richer mixtures with few residuals. The trend is less strong with increasing residual fraction such that the effect of $f$ appears to suppress the effect of $\phi$, with $L_{\mathrm{b}}$ approaching an average value of about $-0.8 \mathrm{~mm}$. However, more data are required to confirm this.

It is informative to compare the present measurements, with those obtained elsewhere. Shown in Fig. 11 is the variation of unstretched laminar burning velocity with equivalence ratio, for hydrogen-air mixtures at normal (atmospheric) pressure and temperature, NTP. The various shaded symbols denote values, measured in this

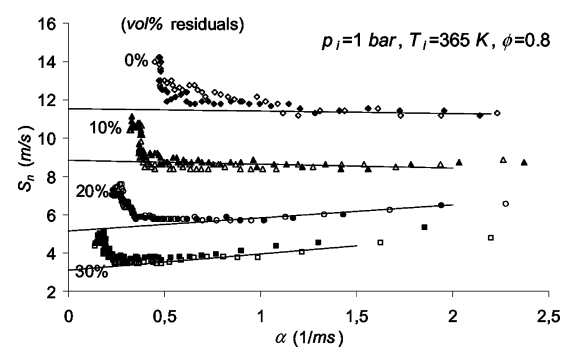

Fig. 8. Flame speed vs. flame stretch rate for flames at $\phi=0.8,365 \mathrm{~K}$, and 1 bar with various residual gas concentrations. 


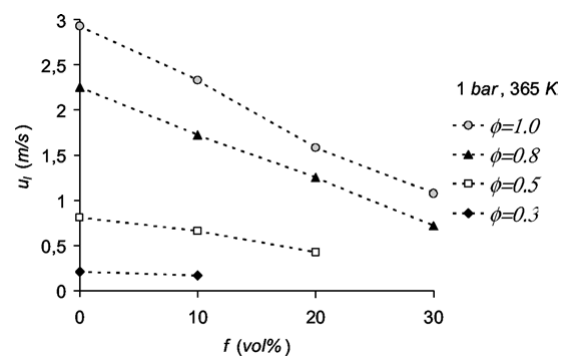

Fig. 9. Effect of residual gas concentration $f$ on burning velocities $u_{1}$, for 1 bar $365 \mathrm{~K}$ flames.

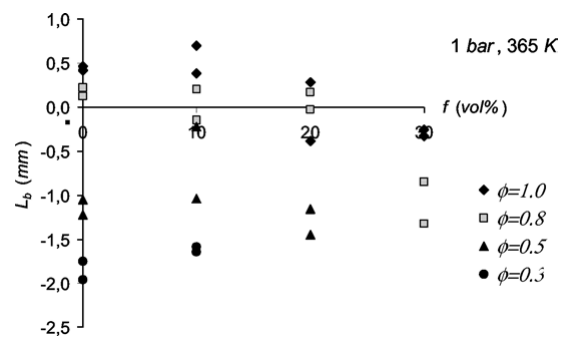

Fig. $10 . L_{\mathrm{b}}$ vs. residual gas concentration $f$, for 1 bar $365 \mathrm{~K}$ hydrogen-air flames.

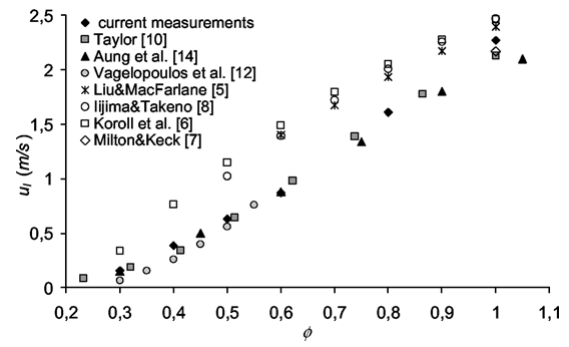

Fig. 11. Laminar burning velocities plotted against equivalence ratio, for NTP $\mathrm{H}_{2}$-air flames.

work, by Taylor [10] and Aung et al. [14] from

\section{spherically expanding flames and by Vagelopou-} los et al. [12] using a counter flow stabilised burner. All these works specifically reported the unstretched burning velocity, and the agreement between their data is good. However, there is less agreement with the open symbols which denote measurements that did not consider the effects of stretch rate, as reported in [5-8]. These data are consistently higher than those that considered stretch rate. Probable reasons for this are that they may have been recorded under the influence of significant stretch rate which, with negative values of $L_{\mathrm{b}}$, causes higher burning velocities. Also, it is likely that cellular flames would exist in some situations, again increasing the burning velocity. Fig. 12 shows the variation of $L_{\mathrm{b}}$ with $\phi$ for

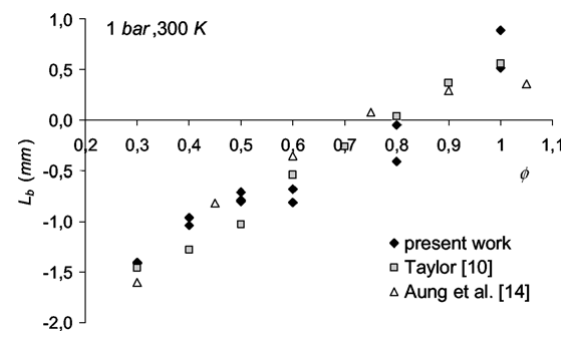

Fig. 12. Burned gas Markstein lengths against equivalence ratio at NTP.

NTP hydrogen-air flames. The Markstein lengths reported in $[10,14]$ were recalculated, by the present authors, to yield $L_{\mathrm{b}}$, according to the definition used in this work and by the methodology described in [18]. Again, good agreement can be seen between the values reported in $[10,14]$ and this work. Lean hydrogen-air mixtures are diffusionally unstable, due to the higher mass diffusivity of the hydrogen molecule compared to the oxygen molecule. Thus, these mixtures show a flame speed reduction with decreasing stretch rate and are characterised by a negative burned gas Markstein length. This also implies an earlier transition to a cellular flame structure as demonstrated here and reported in [22]. As shown in Figs. 10 and 12 and at atmospheric pressure, hydrogen-air flames are diffusionally unstable on the lean side of $\phi \approx 0.8$. For higher pressures, the equivalence ratio below which the Markstein number is negative shifts to richer mixtures [15] which implies that for all practical mixtures in hydrogen engines (stoichiometric to lean and high pressures), the underlying laminar flames are unstable. No significant effect of temperature on the stability was found.

\subsection{Towards a correlation}

For a variety of mixtures, many workers $[7,8,17,19,23]$ have correlated the effects of temperature and pressure on the laminar burning velocity by

$u_{1}=u_{10}\left(\frac{T}{T_{0}}\right)^{\alpha_{\mathrm{T}}}\left(\frac{P}{P_{0}}\right)^{\beta_{\mathrm{p}}}$

Here, the subscript ' 0 ' denotes reference conditions, and the parameters $\alpha_{\mathrm{T}}$ and $\beta_{\mathrm{p}}$ depend on $\phi$. Measurements at various initial temperatures were processed to determine the temperature exponent $\alpha_{\mathrm{T}}$, given by $\log \left(u_{1} / u_{10}\right) / \log \left(T / T_{0}\right)$, and average values at 1 bar are shown in Fig. 13. In general, $\alpha_{\mathrm{T}}$ decreases with $\phi$, although there is considerable scatter. The mean value for $\alpha_{T}$ for the range of $\phi$ between 0.5 and 1 is 1.57 and is comparable with the value of 1.64 re- 


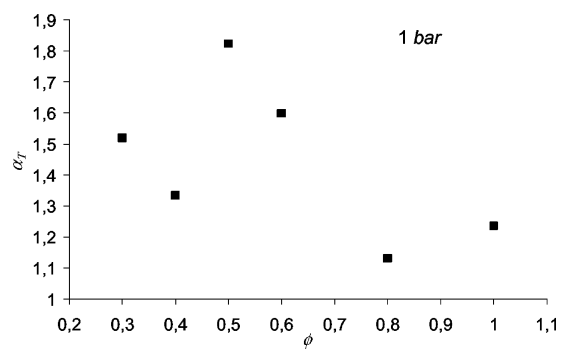

Fig. 13. Temperature exponent $\alpha_{\mathrm{T}}$ vs. equivalence ratio at 1 bar.

ported by Liu and MacFarlane [5] and Koroll et al. [6] over the same range, and with 1.53 reported by Iijima and Takeno [8]. The value for the stoichiometric flame, 1.32, also compares surprisingly well with that by Milton and Keck [7]: 1.26 , who obtained their values from bomb pressure derived data.

As discussed above, at higher pressures, it was not possible to obtain $u_{1}$ or $L_{\mathrm{b}}$ due to the early onset of instabilities. Therefore, the above discussion addresses only values at normal pressure. However, an attempt is made here to address the effect of higher pressure by considering the variation of $u_{n, 10 \mathrm{~mm}}$ with pressure and equivalence ratio. Shown in Fig. 14 is the variation of $u_{n, 10 \mathrm{~mm}}$ with $\phi$ for hydrogen-air mixtures at $365 \mathrm{~K}$ and a range of pressures. Shown in Fig. 15 is the variation of

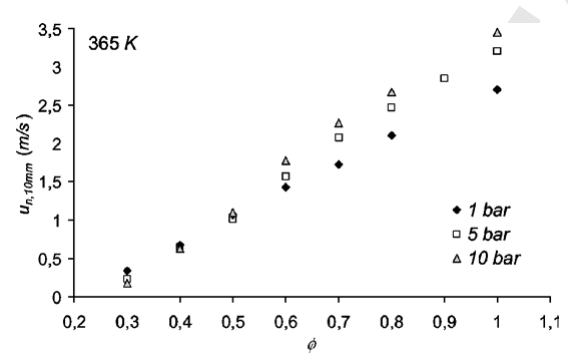

Fig. 14. Burning velocity $u_{n, 10 \mathrm{~mm}}$ vs. equivalence ratio at 1,5 , and 10 bar, $365 \mathrm{~K}$.

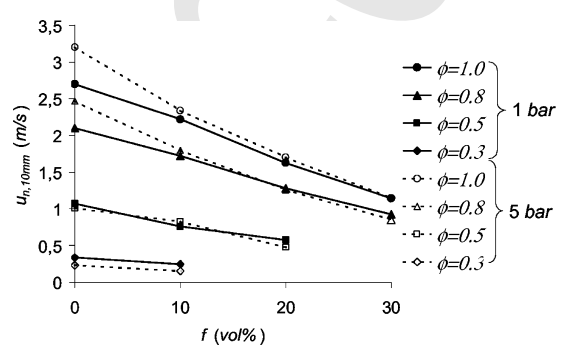

Fig. 15. Effect of residual gas concentration $f$ on burning velocities $u_{n, 10 \mathrm{~mm}}$, for $365 \mathrm{~K}$ flames.

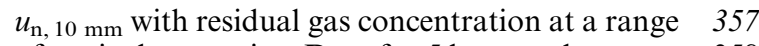
of equivalence ratios. Data for 5 bar are shown, at 358 which $u_{1}$ could not be derived, and values for 1 bar 359 are shown for comparison only since the 360 unstretched data in Fig. 9 are more fundamental 361 and have more general applicability. Figs. 14362 and 15 show that there is an increase in burning 363 velocity with pressure for mixtures on the rich side 364 of about $\phi=0.4$, and decrease in burning velocity 365 with pressure on its lean side. This is consistent 366 with the findings of Lewis and von Elbe [24] 367 who demonstrated a change in the sign of the 368 pressure exponent related to the magnitude of 369 the burning velocity. A linear relationship 370 between burning velocity and residual gas concen- 371 tration is evident in Fig. 15. The influence of resid- 372 uals can thus be described by $u_{1}=u_{10}(1-\gamma f), \quad 373$ where $f$ is the concentration of residuals in volume 374 fraction, and $\gamma$ expresses the influence on burning 375 velocity. There is little influence of pressure on the 376 value of $\gamma$ and it slightly decreases with equiva- 377 lence ratio. The effect of increasing temperature 378 was unclear but seems to decrease $\gamma$. The mean va- 379 lue for $\gamma$ at $365 \mathrm{~K}$ and 1 bar is 2.25. Measurements 380 at various pressures were also obtained at $300 \mathrm{~K} \quad 381$ and they showed similar behaviour to that in 382 Fig. 14. Also, measurements at similar conditions 383 to those in Fig 15 were obtained at $430 \mathrm{~K}$ and 384 showed similar behaviour to that in Fig. 15. It 385 was not possible to repeat the experiments in 386 Fig. 15 at the lower temperature of $300 \mathrm{~K}$ because 387 of condensation of the water content of the simu- 388 lated residuals. Measurements at $\phi=0.25$ were 389 also obtained but these flames were buoyant and 390 were therefore not used in the comparison.

Shown in Fig. 16 is the variation of the pressure exponent, $\beta_{\mathrm{p}}$, which was obtained in the same way as $\alpha_{\mathrm{T}}$ except that $u_{n, 10 \mathrm{~mm}}$ replaced $u_{1}$. The transition from a negative to a positive pressure dependence at $\phi=0.4$ can clearly be seen. Values for $\beta_{\mathrm{p}}$ were also derived from the data published by Aung et al. [15] and are also shown in Fig. 16. They show a similar transition of $\beta_{\mathrm{p}}$ with $\phi$, although at a higher value of the latter and with a stronger, more linear dependency. The value derived from pressure data reported by Milton and Keck [7] is also shown in Fig. 16. It is much higher

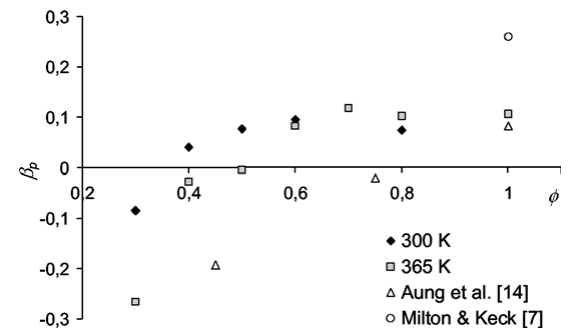

Fig. 16. Pressure exponent $\beta_{\mathrm{p}}$ vs. equivalence ratio for 300 and $365 \mathrm{~K}$. 
than the other, stretch-corrected, data, for reasons discussed above in relation to stretch and cellularity effects.

\section{Conclusions}

Centrally ignited, spherically expanding hydrogen-air flames were measured using schlieren photography to determine the influence of equivalence ratio, initial pressure, temperature, and residuals of combustion on the burning velocity and effects of stretch rate.

An increase in initial temperature was shown to increase the unstretched laminar burning velocity but was shown not to have a significant effect on $L_{\mathrm{b}}$ or flame structure. The temperature exponent $\alpha_{T}$ is of the same order as values reported in other studies even though they did not account for stretch rate.

The effect of pressure is difficult to derive because flames become cellular with increasing pressure. An attempt was made to study this effect by using a burning velocity at a different reference condition to the more generally accepted, unstretched laminar burning velocity with the burning velocity presented for a radius of $10 \mathrm{~mm}$. The influence of pressure was demonstrated at a wide range of conditions. A pressure exponent was obtained which exhibited a crossover from negative to positive values at $\phi \sim 0.4$.

The laminar burning velocity and $u_{n, 10 \mathrm{~mm}}$ were shown to decrease with increasing concentrations of residual gas. A decrease in $L_{\mathrm{b}}$ also was observed.

The results of the measurements reported here will aid the construction of a correlation of hydrogen-air residual burning velocities for use in thermodynamic engine codes for hydrogen-fuelled engines. Unstretched data as a function of temperature and residual gas content have not been previously published.

\section{Acknowledgments}

The authors express their sincere thanks to Prof. Derek Bradley, University of Leeds, for valuable, ongoing discussions, and the E.C. for funding a Marie Curie Fellowship (ENK6-CT2000-57), during which the measurements reported were undertaken.

\section{References}

[1] J.W. Heffel, Int. J. Hydrogen Energy 28 (2003) 451 1285-1292.

[2] S. Verhelst, R. Sierens, Int. J. Hydrogen Energy 26453 (2001) 981-985.

[3] S. Verhelst, R. Sierens, Int. J. Hydrogen Energy 26 (2001) 987-990.

[4] S. Verhelst, R. Sierens, Simulation of hydrogen combustion in spark-ignition engines, in: Proceedings of the 14th World Hydrogen Energy Conference, Montreal, 2002.

[5] D.D.S. Liu, R. MacFarlane, Combust. Flame 49 (1983) 59-71.

[6] G.W. Koroll, R.K. Kumar, E.M. Bowles, Combust. Flame 94 (1993) 330-340.

[7] B.E. Milton, J.C. Keck, Combust. Flame 58 (1) (1984) 13-22.

[8] T. Iijima, T. Takeno, Combust. Flame 65 (1986) 3543.

[9] D.R. Dowdy, D.B. Smith, S.C. Taylor, A. Williams, Proc. Combust. Inst. 23 (1990) 325-332.

[10] S.C. Taylor, Burning velocity and the influence of flame stretch, Ph.D. Thesis, Leeds University, 1990.

[11] F.N. Egolfopoulos, C.K. Law, Proc. Combust. Inst. 23 (1990) 333-340.

[12] C.M. Vagelopoulos, F.N. Egolfopoulos, C.K. Law, Proc. Combust. Inst. 25 (1994) 1341-1347.

[13] S. Kwon, L.K. Tseng, G.M. Faeth, Combust. Flame 90 (1992) 230-246.

[14] K.T. Aung, M.I. Hassan, G.M. Faeth, Combust. Flame 109 (1997) 1-24.

[15] K.T. Aung, M.I. Hassan, G.M. Faeth, Combust. Flame 112 (1998) 1-15.

[16] L. Gillespie, M. Lawes, C.G.W. Sheppard, R. Woolley, in: A.K. Oppenheim, F. Stodolsky (Eds.), Advances in Combustion, Society of Automotive Engineers, ISBN 0-7680-0542-6, 2000, pp. 1-22.

[17] D. Bradley, R.A. Hicks, M. Lawes, C.G.W. Sheppard, R. Woolley, Combust. Flame 115 (1-2) (1998) 126-144.

[18] D. Bradley, P.H. Gaskell, X.J. Gu, Combust. Flame 104 (1-2) (1996) 176-198.

[19] X.J. Gu, M.Z. Haq, M. Lawes, R. Woolley, Combust. Flame 121 (2000) 41-58.

20] S.D. Tse, D.L. Zhu, C.K. Law, Proc. Combust. Inst. 28 (2000) 1793-1800.

[21] O.C Kwon, G.M. Faeth, Combust. Flame 124 (2001) 590-610.

[22] D. Bradley, C.G.W. Sheppard, R. Woolley, D.A. Greenhalgh, R.D. Lockett, Combust. Flame 122 (2000) 195-209.

[23] M. Metghalchi, J.C. Keck, Combust Flame 48 (1982) 191-210.

[24] B. Lewis, G. von Elbe, Combustion, Flames and Explosions of Gases, third ed., Academic Press, New York, London, 1987. 